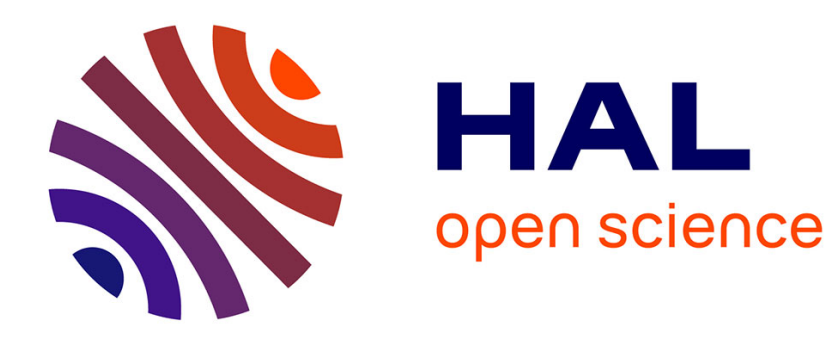

\title{
Onde thermique dans l'hélium 4 cubique centré
}

B. Castaing, A. Libchaber

\section{To cite this version:}

B. Castaing, A. Libchaber. Onde thermique dans l'hélium 4 cubique centré. Journal de Physique Lettres, 1975, 36 (12), pp.309-311. 10.1051/jphyslet:019750036012030900 . jpa-00231216

\section{HAL Id: jpa-00231216 https://hal.science/jpa-00231216}

Submitted on 1 Jan 1975

HAL is a multi-disciplinary open access archive for the deposit and dissemination of scientific research documents, whether they are published or not. The documents may come from teaching and research institutions in France or abroad, or from public or private research centers.
L'archive ouverte pluridisciplinaire HAL, est destinée au dépôt et à la diffusion de documents scientifiques de niveau recherche, publiés ou non, émanant des établissements d'enseignement et de recherche français ou étrangers, des laboratoires publics ou privés. 


\title{
ONDE THERMIQUE DANS L'HÉLIUM 4 CUBIQUE CENTRÉ
}

\author{
B. CASTAING et A. LIBCHABER \\ Groupe de Physique des Solides de l'Ecole Normale Supérieure, \\ 24, rue Lhomond, 75231 Paris Cedex 05, France
}

(Rę̧u le 28 juillet 1975, accepté le 12 septembre 1975)

\begin{abstract}
Résumé. - Dans l'hélium 4 cubique centré, entre $1,4 \mathrm{~K}$ et $1,8 \mathrm{~K}$, et près de la transition solideliquide, nous observons la propagation d'une impulsion de chaleur, qu'il est difficile de relier théoriquement à un mode de deuxième son.

Abstract. - In body centered cubic helium 4 between $1.4 \mathrm{~K}$ and $1.8 \mathrm{~K}$ and very close to the solidliquid transition, we observe the propagation of a heat pulse of velocity about $100 \mathrm{~m} / \mathrm{s}$. It is difficult to relate it to the propagation of a second sound wave because of possible Umklapp processes. We propose various possible mechanisms for this effect.
\end{abstract}

1. Introduction. - Dans cette lettre nous présentons des résultats préliminaires sur une expérience de propagation d'impulsions de chaleur dans l'hélium 4 cubique centré, où nous observons la propagation d'une onde de température.

La phase cubique centrée de l'hélium 4 solide présente de nombreuses anomalies. En particulier sa conductivité thermique est basse et certains phonons de bord de zone ont une durée de vie très courte $[1,2]$. Ces anomalies pourraient être liées aux propriétés particulières des lacunes dans cette phase, qui ont une énergie de formation très faible et pourraient se propager librement dans le cristal $[3,4]$. D'un autre point de vue certains auteurs ont examiné la possibilité d'existence d'une phase solide possédant le même type d'ordre que le superfluide en plus de l'ordre cristallin [5]. La place de cette nouvelle phase dans le diagramme pression-température serait proche de celle occupée par la phase cubique centrée. Aucune évidence expérimentale ne semble, pour l'instant, confirmer ce modèle.

Dans notre expérience une impulsion de chaleur se propage dans un cristal cubique centré. La seule onde de température observée dans un solide est le deuxième son. Dans les théories du second son une variation de la densité d'excitations du type phonon se propage comme une onde atténuée [6]. Cette atténuation est faible lorsque les fréquences de phonons se trouvent dans la fenêtre $\tau_{\mathrm{N}} \ll \omega^{-1} \ll \tau_{\mathrm{R}}$ où $\tau_{\mathrm{N}}$ et $\tau_{\mathrm{R}}$ sont les temps de relaxation respectifs des processus phonon-phonon normaux et résistifs. Dans l'hélium 4 les effets résistifs essentiels sont causés par les processus Umklapp [6]. La température de Debye de la phase cubique centrée étant très faible, $\theta_{\mathrm{D}} \simeq 25 \mathrm{~K}$, l'onde de second son devrait être très fortement atténuée dans la gamme de température d'existence de la phase cubique centrée $1,4 \mathrm{~K}<T<1,8 \mathrm{~K}$.

2. Le dispositif expérimental. - L'hélium étudié est situé dans un volume qui peut varier sous l'action d'un piston déformant un soufflet. Ce piston est actionné par l'extension d'un autre soufflet de section supérieure contenant de l'hélium à pression variable. L'hélium est introduit par le bas dans la chambre d'expérience, venant d'une bouteille sous pression munie d'un détendeur. Quand le fluide se solidifie dans le tuyau d'amenée, isolant le volume étudié, l'hélium contenu dans celui-ci est encore liquide mais à la pression de solidification. Le piston permet alors de solidifier progressivement cet hélium et d'augmenter ensuite la pression dans le cristal ainsi formé.

L'impulsion de chaleur est émise et détectée par des résistances à couche Cermet [7], dont les dimensions sont réduites à $1 \mathrm{~mm}^{2}$ environ. La résistance de l'émetteur est de $50 \Omega$ celle du récepteur $50 \mathrm{k} \Omega$. Les impulsions électriques sont formées à partir d'un générateur H.P. 214A; le signal de réception est amplifié par un tiroir Tektronix 1A7A suivi d'un Boxcar P.A.R. Emetteur et récepteur sont situés à $6 \mathrm{~mm}$ l'un de l'autre dans un plan horizontal. Les échos d'un quartz longitudinal nous permettent de détecter séparément les transitions entre la phase liquide et les phases solides.

3. L'expérience. - Nous nous sommes placés à $1,6 \mathrm{~K}$, milieu du domaine d'existence en température de la phase cubique centrée et nous avons comprimé progressivement l'hélium à température constante 
au voisinage de la pression de solidification. Nous avons vu disparaitre le signal de second son dans le liquide en même temps qu'apparaît un nouveau signal à plus grande vitesse suivi d'un écho (Fig. 1). Alors que la vitesse du second son dans le liquide à cette température et à cette pression est de $12,5 \mathrm{~m} / \mathrm{s}$, la vitesse du nouveau signal est d'environ $100 \mathrm{~m} / \mathrm{s}$. $\mathrm{Si}$ l'on continue à réduire le volume, le signal se modifie régulièrement et finit par disparaître. Parallèlement les échos de quartz disparaissent puis réapparaissent en passant du liquide au solide indiquant un changement dans la vitesse du son. Ce changement correspond à celui attendu au passage $\mathrm{He}$ liquide- $\mathrm{He}$ cubique centré. Lorsque le nouveau signal disparaît, les échos disparaissent aussi. Ceci correspond sans doute au fait que la transition cubique centréehexagonale compacte est trop brutale pour que nous obtenions un monocristal.

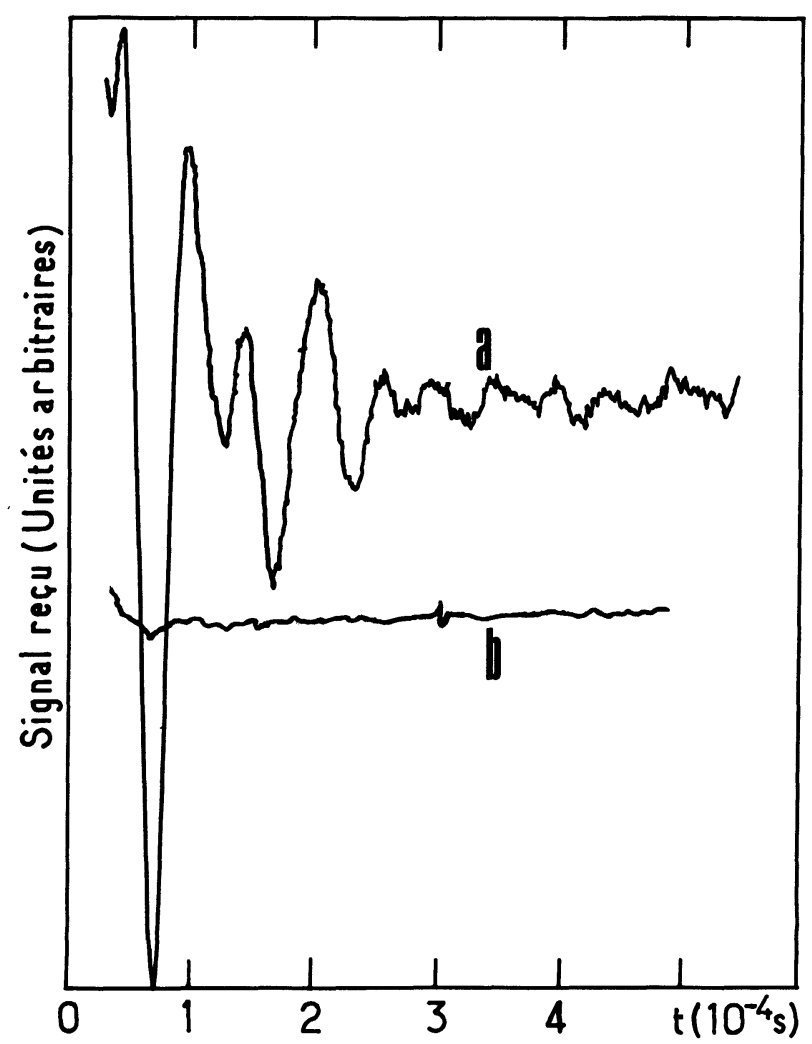

Fig. 1. - a) Observation du nouveau signal et d'un écho dans l'hélium 4 cubique centré; $T=1,6 \mathrm{~K}$, distance émetteur-récepteur $6 \mathrm{~mm}$, pression de solidification; $b$ ) Absence de signal pour $T=0,9 \mathrm{~K}$, pression de solidification.

Nous avons répété cette expérience à différentes températures. Nous observons la propagation de ce nouveau signal dans le solide à la pression de solidification entre $1,4 \mathrm{~K}$ et $1,8 \mathrm{~K}$. En diminuant la température à partir de $1,4 \mathrm{~K}$, nous voyons apparaître un signal de grande amplitude instable dû sans doute à la transition cubique centrée-hexagonale compacte. Puis le signal disparait. Vers $1 \mathrm{~K}$ nous voyons apparaitre la diffusion thermique, puis vers $0,7 \mathrm{~K}$ le second son ordinaire, très bien défini, de la phase hexagonale compacte suivie de deux échos (Fig. 2).

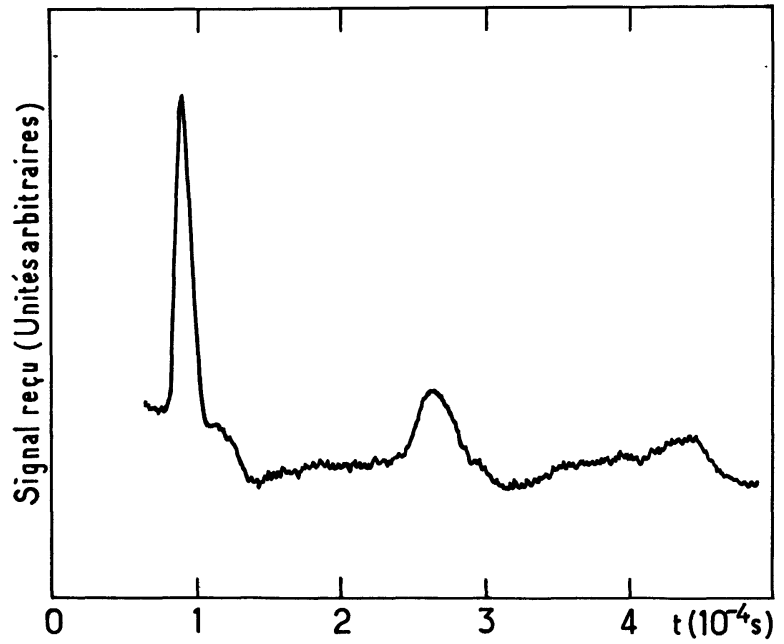

Fig. 2. - Deuxième son dans l'hélium 4 hexagonal compact : signal suivi de deux échos. $T=0,5 \mathrm{~K}$; distance émetteur-récepteur $11,5 \mathrm{~mm}$, pression de solidification.

Nous avons également répété l'expérience avec deux paires émetteur-récepteur à propagation horizontale situées à environ $1 \mathrm{~mm}$ l'une au-dessus de l'autre. Quand on déplace l'interface liquide-solide du bas vers le haut, le signal sur la paire du bas (dans le solide) commence par augmenter, puis diminue pour disparaître à peu près au moment où l'interface atteint la paire supérieure émetteur-récepteur. Ceci semble montrer que ce signal n'existe qu'au voisinage de l'interface liquide-solide.

4. Discussion. - Il existe à priori quatre modes de propagation d'une onde de température dans le solide : une onde de surface qui peut être soit thermique soit mécanique, le son ordinaire en volume, le second son, et enfin la propagation d'une zone fondue.

Le second son dans les solides n'existe que si le pseudo-moment total du gaz de phonon est conservé dans les collisions entre phonons. Il est donc impossible dès l'apparition des processus Umklapp, Ackerman et Guyer [6] donnent un critère semi-empirique pour l'apparition du second son. La température doit être inférieure à $\theta / 40$ où $\theta$ est la température de Debye du solide. Ce critère donne $0,8 \mathrm{~K}$ pour la température d'apparition du second son dans l'hélium hexagonal compact, valeur qui correspond bien à nos observations. Dans la phase cubique centrée, la température de Debye est plus faible encore (environ $25 \mathrm{~K}$ ). Le second son ne devrait donc pas être possible dans le domaine d'existence de cette phase. Néanmoins la vitesse observée, $V_{\mathrm{s}} \simeq 100 \mathrm{~m} / \mathrm{s}$, correspond bien à une vitesse typique de deuxième son. Nous avons. examiné diverses raisons pouvant rendre les processus Umklapp moins efficaces, comme le recul de la région du cristal intéressée. Ses dimensions sont de l'ordre du libre parcours des phonons, sa masse $M$ est donc relativement faible et l'énergie de recul $h^{2} G^{2} / 2 M$, où $G$ est le vecteur du réseau réciproque en question, n'est plus négligeable. Mais cela ne réduit que fai- 
blement l'impulsion perdue par le gaz de phonons, nous ne pensons donc pas pouvoir associer ce signal à un deuxième son classique. En fait les processus Umklapp, ou les défauts du cristal, couplent le gaz de phonons avec le cristal parfait, état fondamental du solide. Quand le couplage est fort (mauvaise conduction thermique), l'onde mécanique et l'onde thermique forment une seule et même onde : le son normal adiabatique. On peut donc penser que notre impulsion de chaleur excite directement une onde sonore. Nous pouvons relier l'amplitude de température dans une onde sonore $\delta T_{\mathrm{r}}$ à l'amplitude de température nécessaire pour exciter cette onde par dilatation thermique : $\delta T_{\mathrm{e}}$; on a à peu près :

$$
\frac{\delta T_{\mathrm{r}}}{\delta T_{\mathrm{e}}}=\frac{T \gamma^{2}\left(K C_{\mathrm{v}} \omega\right)^{1 / 2}}{4 \rho c^{3}}=x
$$

où $\gamma$ est la constante de Gruneisen, $K$ la conductivité thermique, $C_{\mathrm{v}}$ la chaleur spécifique, $\rho$ la densité, $c$ la vitesse du son, $T$ la température et $\omega$ la fréquence excitatrice. Dans les conditions normales pour la phase cubique centrée $\left(K \simeq 10^{-1} \mathrm{~W} / \mathrm{m} \mathrm{K}, C_{\mathrm{v}} \simeq 10^{5} \mathrm{~J} /\right.$ $\left.\mathrm{K} \mathrm{m}^{3}, \rho \sim 200 \mathrm{~kg} / \mathrm{m}^{3}, c \simeq 500 \mathrm{~m} / \mathrm{s}, \gamma \simeq 3, \omega \simeq 10^{6}\right)$, $x$ est égal à $10^{-4}$ ce qui rend cette onde inobservable thermiquement. Mais, au voisinage de la transition, dans l'hypothèse d'un mode mou dont la vitesse tomberait à $100 \mathrm{~m} / \mathrm{s}$ (vitesse approximative du signal), et une valeur de $\gamma$ correspondante de l'ordre de 50, $x$ devient de l'ordre de $10^{-1}$, ce qui rend bien compte de l'amplitude du signal par rapport à celle du second son dans le solide. Cette explication est cohérente avec le fait que le signal n'existe qu'au voisinage de la surface. Mais un tel mode mou n'a pas été observé.

L'hypothèse d'une onde de surface expliquerait également que le signal n'existe qu'au voisinage de la surface. Mais une onde de surface thermique nous semble devoir se propager beaucoup plus lentement. En effet, en l'absence de tout mode de propagation de la chaleur dans le solide, l'onde thermique de surface devrait ressembler au second son dans le liquide, légèrement modifiée par la présence du solide. $\mathrm{Sa}$ vitesse ne pourrait donc pas être très différente de celle du second son dans le liquide $(10 \mathrm{~m} / \mathrm{s}$ au lieu des $100 \mathrm{~m} / \mathrm{s}$ observées). D'autre part une onde de surface mécanique (onde de Rayleigh) est faiblement couplée au récepteur. Elle serait détectée, de même qu'une onde de son ordinaire, par les fluctuations de température associées aux fluctuations de pression. Une onde de surface ne semble donc pas être une explication satisfaisante.

Une dernière possibilité pourrait être la propagation d'une zone fondue à partir de l'émetteur. Ce serait l'analogue d'une onde de choc. Une telle discontinuité semble avoir deux possibilités de propagation, l'une acoustique de vitesse $u_{1}=\frac{\rho}{\rho^{\prime}} c^{\prime}$, où $\rho^{\prime}$ est la densité du liquide et $c^{\prime}$ la vitesse du son dans le liquide, l'autre thermique de vitesse $u_{2}=v_{\mathrm{II}} \frac{\rho}{\rho^{\prime}} \sqrt{\frac{\sigma}{\sigma^{\prime}}}$ où $\sigma$ et $\sigma^{\prime}$ sont les entropies par unité de masse du solide et du liquide et $v_{\text {II }}$ la vitesse du second son dans le liquide. Aucune de ces deux vitesses ne correspond à celle de notre signal.

En conclusion l'explication la plus satisfaisante semble peut-être l'hypothèse d'une variation rapide des constantes élastiques au voisinage de la transition solide-liquide (mode mou). Ce mode mou pourrait n'exister que dans certaines directions cristallographiques et cela pourrait expliquer que le signal n'apparaisse pas toujours à la solidification. Mais il reste à observer l'onde sonore associée.

Finalement nous ne pouvons exclure l'hypothèse d'une onde de deuxième son si la possibilité de superfluidité de la phase cubique centrée était expérimentalement vérifiée. De toute manière toute explication suppose que la phase cubique centrée près de la transition liquide est fortement anormale.

\section{Bibliographie}

[1] GUYeR, R. A., Solid State Phys. 23 (Academic Press) 1969.

[2] Minkiewicz, V. J., Kitchens, T. A., Shirane, G., Osgood, F. B., Phys. Rev. A 8 (1973) 1513.

[3] ANDReEv, A. F., LifShitZ, I. M., JETP 29 (1969) 1107.
[4] Sullivan, N., Deville, G., Landesman, A., Phys. Rev. B 11 (1975) 1858

[5] Liu, K., FisheR, M. E., J. Low Temp. Phys. 10 (1973) 655.

[6] Ackerman, C. C., Guyer, R. A., Ann. Phys. 50 (1968) 128.

[7] Fabriqué à partir de potentiomètres Sfernice. 